\title{
Correction to: ZGLS: a novel flat quorum-based and reliable location management protocol for VANETs
}

\author{
Maaz Rehan ${ }^{1,2} \cdot$ Halabi Hasbullah ${ }^{1} \cdot$ Ibrahima Faye $^{3} \cdot$ Waqas Rehan $^{4} \cdot$ Omer Chughtai $^{5,6} \cdot$ \\ Mubashir Husain Rehmani ${ }^{6}$
}

Published online: 28 August 2018

(C) Springer Science+Business Media, LLC, part of Springer Nature 2018

\section{Correction to: Wireless Netw (2018) 24:1885-1903}

https://doi.org/10.1007/s11276-016-1443-4

The original version of this article contained an error in coauthor's affiliation. The correct affiliation of Dr. Waqas Rehan is given in this erratum.

The original article can be found online at https:// doi.org/10.1007/s11276-016-1443-4.

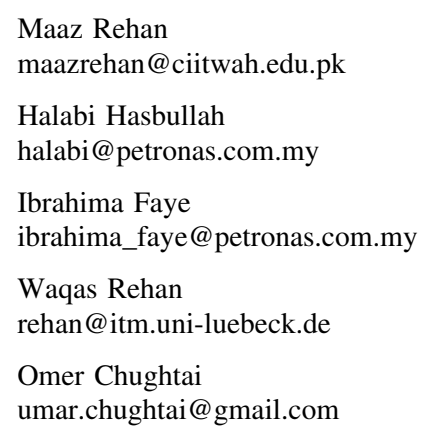

1 Department of Computer and Information Sciences, Universiti Teknologi PETRONAS, 32610 Perak, Malaysia
2 Department of Computer Science, COMSATS Institute of Information Technology, Wah Cantt, Pakistan

3 Department of Fundamental and Applied Sciences, Universiti Teknologi PETRONAS, 32610 Perak, Malaysia

4 Institute of Telematics, University of Lübeck, 23562 Lübeck, Germany

5 Department of Electrical and Electronic Engineering, Universiti Teknologi PETRONAS, 32610 Perak, Malaysia

6 Department of Electrical Engineering, COMSATS Institute of Information Technology, Wah Cantt, Pakistan 